\title{
Laboreal
}

Volume $17 \mathrm{~N}^{\circ} 2$ | 2021

Trabalho e Território

\section{Horarios atípicos de trabajo y temporalidades humanas : la necesidad de un enfoque interdisciplinario en ergonomía}

Tempos de trabalho atípicos e temporalidades humanas : a necessidade de uma abordagem interdisciplinar em ergonomia

Temps de travail atypiques et temporalités humaines: de l'exigence d'une approche interdisciplinaire en ergonomie

Atypical working times and human temporalities: the requirement for an interdisciplinary approach in ergonomics

\section{Sophie Prunier-Poulmaire y Béatrice Barthe}

\section{OpenEdition}

\section{Journals}

Edición electrónica

URL: https://journals.openedition.org/laboreal/18120

DOI: $10.4000 /$ laboreal. 18120

ISSN: 1646-5237

Editor

Universidade do Porto

Referencia electrónica

Sophie Prunier-Poulmaire y Béatrice Barthe, «Horarios atípicos de trabajo y temporalidades humanas la necesidad de un enfoque interdisciplinario en ergonomía», Laboreal [En línea], Volume 17 №2 | 2021, Publicado el 01 diciembre 2021, consultado el 05 diciembre 2021. URL: http:// journals.openedition.org/laboreal/18120 ; DOl: https://doi.org/10.4000/laboreal.18120

Este documento fue generado automáticamente el 5 diciembre 2021

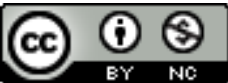

Laboreal está licenciado com uma Licença Creative Commons - Atribuição-NãoComercial 4.0 Internacional. 


\section{Horarios atípicos de trabajo y temporalidades humanas : la necesidad de un enfoque interdisciplinario en ergonomía}

Tempos de trabalho atípicos e temporalidades humanas : a necessidade de uma abordagem interdisciplinar em ergonomia

Temps de travail atypiques et temporalités humaines: de l'exigence d'une approche interdisciplinaire en ergonomie Atypical working times and human temporalities: the requirement for an interdisciplinary approach in ergonomics

Sophie Prunier-Poulmaire y Béatrice Barthe

\section{NOTA DEL EDITOR}

Traducido por : Gabriela Cuenca (gcuenca@frba.utn.edu.ar)

Manuscrito recibido en : 26/07/2021

Aceptado tras peritaje : 30/09/2021

\section{Un enfoque prospectivo a escala de vida}

1 El texto de Charles Gadbois apareció hace casi 30 años en las Actas del coloquio "Recherches pour l'ergonomie", celebrado en 1993 en la Universidade de Toulouse Jean Jaurès (Recherches pour l'ergonomie, 1993). Estas primeras jornadas dedicadas a la investigación en la disciplina fueron ideadas por Yvon Quéinnec, colega y amigo de Charles Gadbois. Yvon Quéinnec deseaba crear en Toulouse el equivalente a las primeras Journées de la Pratique de l'ergonomie, iniciadas ese mismo año en Burdeos, 
por François Daniellou y el Laboratoire d'Ergonomie des Systèmes Complexes de l'Institut National Polytechnique. La segunda y última edición de las jornadas de Toulouse tuvo lugar cinco años después, en 1998 y se denominó "Recherche et ergonomie, 1998". Este cambio de preposición (pour/et - para/y) en la forma de pensar el lugar y el rol de la ergonomía en la producción de conocimiento científico no es, para nada, menor. Al final, estas jornadas de investigación para/y/en ergonomía no perduraron, lo que quizás refleje la dificultad que tuvo la comunidad en ese momento, en lograr consenso para reconocer a la ergonomía como una disciplina científica por derecho propio.

2 Sin embargo, en este breve texto de Charles Gadbois, que ha permanecido confidencial, se trata efectivamente de una cuestión de investigación en ergonomía. Destacado especialista sobre el tiempo de trabajo, de los horarios alternados al trabajo nocturno y por turnos, dedicó su carrera a comprender en profundidad los efectos de estos horarios atípicos de trabajo y fuera del estándar, que él definía como "horarios que imponen un marco temporal de trabajo en discordancia con la vida cotidiana del ser humano" (Gadbois, 1993, p. 183, traducción libre). Su intervención en estas jornadas dedicadas a la investigación ergonómica le brinda la oportunidad de proponer, con visión de futuro, líneas de trabajo para desarrollar la investigación sobre este tema complejo y en rápida expansión, en lugar de hacer un balance del estado de los conocimientos científicos sobre los horarios atípicos de trabajo.

3 Partiendo de la constatación de que existe un desconocimiento de los numerosos problemas que plantea el trabajo en horarios atípicos, Charles Gadbois defiende en este texto la importancia de (1) convocar y articular varias disciplinas para abordar este problema y (2) diseñar nuevos modelos que integren el modelo ergonómico de la actividad, que él mismo adaptó con Yvon Quéinnec en trabajos realizados sobre el 3x8h en la industria (Gadbois \& Quéinnec, 1984). En este texto, se refiere a la asociación internacional "Working Time Society", existente desde 1957 e inicialmente denominada " Night and Shiftwork", que sigue siendo hoy una red de científicos y actores clave en este tema. Se lamenta que el conjunto de trabajos sobre el trabajo nocturno y por turnos realizado dentro de esta reconocida red internacional (principalmente en biología, psicología y sociología) se limite a enfoques mono-disciplinarios y, por lo tanto, necesariamente simplificados.

Charles Gadbois también lamenta que exista tal compartimentación en los trabajos provenientes de la psicología social y la sociología, en particular en los modelos propuestos sobre la vida laboral y extra-laboral, tema del cual rápidamente se constituye en uno de los principales expertos franceses. A este respecto, menciona la fructífera contribución del modelo " Système des activités" de Curie y Hajjar (1987) y la necesidad de crear las condiciones que favorezcan el desarrollo de esta perspectiva teórica. Así, abriendo camino, ha conseguido a lo largo de su carrera conciliar, los enfoques de la psicología social, la psicología del trabajo y la ergonomía enriqueciéndolos, sin desvirtuarlos. Así mismo propondrá considerar las cuestiones relativas a los marcos temporales desde un punto de vista diacrónico, es decir, a nivel de vida, en la línea con los trabajos iniciados por Antoine Laville y Serge Volkoff sobre el envejecimiento y las condiciones de trabajo (Volkoff, Laville, \& Maillard, 1992). 


\section{Charles Gadbois, un precursor}

5 Charles Gadbois nació en 1937. Luego de formarse en psicología, se incorpora al Centre national de la recherche scientifique (CNRS), en octubre de 1968 convirtiéndose en Director de Investigación. Colabora con Jacques Leplat, Antoine Laville, Annie WeillFassina y Alain Kerguelen mientras trabaja en el Laboratoire d'Ergonomie Physiologique et Cognitive de l'École Pratique des Hautes Etudes. En 2007, es nombrado miembro honorario de la Societé d'Ergonomie de Langue Francaise (SELF), lo que demuestra su compromiso con la evolución y el desarrollo de la disciplina. A lo largo de su carrera, ha trabajado para que se reconozca la singularidad de las condiciones de trabajo en el sector hospitalario : desde la especificidad de las exigencias que allí se plantean hasta la contundencia de los riesgos que allí aparecen. Su trabajo ha dejado huella en la comunidad, tanto por los conocimientos que ha aportado sobre el propio trabajo hospitalario como sobre el trabajo por turnos y nocturno. Ha logrado imponer una sutil forma de abordar los problemas de este sector de actividad, resaltar las características comunes, útiles para el intercambio de conocimientos dentro de la comunidad científica internacional, teniendo la preocupación permanente de avanzar favorablemente en las situaciones locales. Así, ha demostrado constantemente esta preocupación por mantener un fuerte vínculo con las cuestiones que se plantean en el campo, al tiempo que desarrolla cuestiones teóricas que contribuyen al avance de los conocimientos fundamentales. También es importante destacar el carácter vanguardista de las ideas de Charles Gadbois : su concepción del trabajo hospitalario, las exigencias asociadas a él, los problemas sociales que los afectan, ahora son reavivados cruelmente por la pandemia global de Covid-19. Anticipadamente, supo honrar el carácter excepcional del compromiso del personal hospitalario, alertarnos sobre las desviaciones presentes en el trabajo y sobre los riesgos en que incurriríamos si no los tuviéramos en cuenta.

6 Más allá de este sector, sus conocimientos científicos sobre el trabajo en horarios atípicos se han sedimentado y sus enfoques metodológicos enriquecido gracias a los numerosos sectores profesionales en los que ha intervenido. En su opinión, los imperativos económicos y los provenientes de las exigencias técnicas de los sistemas socio-técnicos han sido factores que impulsaron la difusión y el desarrollo de horarios atípicos.

7 Esta observación, realizada en los años 90, sigue siendo válida hoy en día, aunque hay que añadir los efectos multiplicadores de la globalización, que ha dado lugar a un mundo en constante movimiento, que funciona $24 \mathrm{hs} / 24,7$ días sobre 7 . Hoy en día, el tiempo de trabajo parece estar más condicionado que nunca por estos imperativos ineludibles.

8 Luego de la escritura de este artículo, las imposiciones industriales han invadido progresivamente el sector terciario : también hemos asistido a una amplificación de las exigencias temporales y de los horarios atípicos del sector industrial hacia el sector de servicios, servicios distintos a los intrínsecamente vinculados a este tipo de organización (como hospitales, defensa, justicia, policía, servicio penitenciario, etc.) (Prunier-Poulmaire \& Gaudart, 2018). Así, el trabajo en horarios atípicos parece, más que antes, ir de la mano de una densificación del trabajo. Las nuevas formas de organización parecen aglutinar diferentes temporalidades que chocan entre sí, lo que 
se traduce en una acumulación de exigencias profesionales que acaban afectando la salud y la vida personal de los trabajadores.

El panorama de los horarios atípicos de trabajo es también el resultado de los cambios en la legislación, que ha ido pasando progresivamente de la norma general a las especificidades de las negociaciones locales. Así, a partir de los años ochenta, con una aceleración a raíz de la publicación del texto de Charles Gadbois, asistimos a lo que Thoemmes (2000) llama el "fin del tiempo de trabajo" con la desaparición de un único tiempo de trabajo para todos. La producción del derecho se desplaza del Estado a las empresas y establecimientos, a través de la negociación colectiva. Las decisiones sobre los horarios de trabajo ya no se basan en la mejora de las condiciones de trabajo de los trabajadores, sino en la adaptación a las variaciones del mercado. Esta nueva gestión del tiempo va acompañada de una gran diversificación de estos tiempos negociados, con una explosión de tiempos de trabajo individuales y singulares constituidos por horarios y duraciones atípicas.

Si bien Charles Gadbois fue un precursor en este campo, el sector hospitalario siempre ha sido objeto de una atención especial en el ámbito de la investigación científica en ergonomía como en otras disciplinas. Desde 2020, la pandemia ha aumentado la exigencia de trabajos en el sector hospitalario y ha exacerbado la urgencia de abordar las condiciones de trabajo del personal sanitario,-y en particular las cuestiones de tiempo que estructuran su vida profesional y dan forma a su vida personal. Además, el teletrabajo, brutalmente generalizado durante la crisis sanitaria de 2020, ha hecho superponer el tiempo y el espacio en un único asunto : el lugar de trabajo ha ingresado en los hogares de los trabajadores y el tiempo profesional se ha deslizado en la temporalidad familiar mucho más que antes, relanzando así la necesidad de una investigación detallada que arroje luz sobre los límites entre la vida en el trabajo y la vida fuera del trabajo. Por último, esta catástrofe sanitaria mundial ha puesto de manifiesto numerosas ocupaciones, calificadas como vitales en esta ocasión, para las que el trabajo ininterrumpido persiste y se incrementa en el tiempo, imponiendo a todos, un horario profesional que entra en conflicto con los ritmos biológicos y sociales comunes del ser humano. Aquí se originan los problemas de salud y las dificultades familiares y sociales.

11 Sin embargo, el desconocimiento sobre los problemas que plantean los horarios atípicos, que se lamentaba Charles Gadbois, 30 años después, siguen vigentes.

12 Por lo tanto, cabe preguntarse por qué el impulso dado en su momento no permitió el desarrollo de trabajos en este campo específico, cuando no han dejado de cristalizar a lo largo de los años y en todo el mundo, fuertes intereses económicos, sociales, societarios y políticos. La dificultad reside sin duda en el hecho de que el tiempo de trabajo, único factor común a todas las situaciones profesionales, sigue siendo un tema de investigación complejo debido a su constante evolución. La racionalización económica del tiempo de trabajo, su desregulación progresiva y la multiplicación de los sectores profesionales afectados lo han convertido en un objeto de estudio en constante cambio. Así, en una década hemos asistido a la multiplicación de las formas horarias de trabajo por turnos y a la aparición de una infinidad de horarios cada vez más atípicos, lo que complejiza su análisis.

13 Además, como sucede en todo el mundo, la situación socio-familiar de los franceses ha cambiado considerablemente en los últimos años. Las características de la población, y en particular la situación familiar, siempre han sido objeto de un análisis minucioso ya 
que definen los efectos de los horarios de trabajo por turnos, en la salud y en la unidad socio-familiar. Hoy en día, la multiplicidad de configuraciones familiares (divorcios, segundas nupcias, células monoparentales, etc.) hace que estos análisis sean considerablemente más complejos. Así, al mismo tiempo que los horarios diurnos regulares daban paso a los atípicos, la familia tradicional cambiaba. Estos dos fenómenos no sólo se han combinado, sino que sus efectos se han potenciado. En consecuencia, la regulación de las exigencias de los horarios atípicos por parte de los trabajadores se ha vuelto más incierta y costosa.

14 A pesar de esta complejidad, la investigación debe continuar para esclarecer los cambios organizativos temporales a fin de preservar la salud y la vida fuera del trabajo de los trabajadores. Esto requiere, como planteaba Charles Gadbois, una investigación científica que, aunque arraigada en diferentes campos disciplinarios, produzca conocimientos que converjan y se articulen. En este enfoque interdisciplinario el de la ergonomía.es fundamental.

\section{Un enfoque de los tiempos de trabajo necesariamente interdisciplinario}

15 En efecto, la ergonomía es una ciencia interdisciplinaria que produce una mirada específica y nueva sobre el trabajo $\mathrm{y}$, más precisamente, sobre la actividad de hombres y mujeres en el trabajo, sus determinantes y sus consecuencias. Es lo que defiende Charles Gadbois en este texto y en el conjunto de su producción científica y lo que ha defendido a través de su práctica de investigador, lo más cerca posible a las situaciones de trabajo.

16 Los horarios de trabajo por turnos y nocturnos como objeto de investigación interpelan a muchas disciplinas científicas, donde cada una aporta respuestas particulares y específicas (Costa, Barthe, \& Prunier-Poulmaire, 2013). Estos conocimientos proceden, por ejemplo, de la demografía, la epidemiología, la cronobiología, la crono-psicología, la fisiología, la medicina del trabajo, la neurobiología, la psicología, la sociología, la historia e incluso la geografía, donde, cada una esclarece un aspecto de este objeto de investigación.

17 Pensar en la interdisciplinariedad es integrar estos diferentes enfoques para producir nuevos conocimientos, nuevos y más relevantes marcos de análisis teóricos y metodológicos que van mediante la articulación y la confrontación de diversos tipos de conocimientos mucho más allá de la acumulación de conocimientos disciplinarios. La interdisciplinariedad también permite llegar a acciones coherentes y eficaces para abordar la cuestión de la organización y el diseño de los horarios atípicos de trabajo (Barthe, 2016).

18 Esta exigencia de interdisciplinariedad se plasma en el enfoque de la Agence nationale de sécurité sanitaire de l'alimentation, de l'environnement et du travail (ANSES), cuyas misiones abarcan la evaluación de riesgos en el ámbito de la alimentación, el medio ambiente y el trabajo, con el fin de orientar a los poderes públicos en su política sanitaria. De 2012 a 2016, participamos con dieciocho expertos internacionales (cronobiólogos, epidemiólogos, neurobiólogos, médicos, oncólogos, prevencionistas, médicos del trabajo y ergónomos) en un peritaje colectivo sobre los riesgos sanitarios relacionados con el trabajo en horarios atípicos, especialmente de noche. El informe se 
hizo público el 22 de junio de 2016 (ANSES, 2016). Ante la necesidad de evaluar los efectos de los horarios atípicos en toda su diversidad, continuamos nuestra participación en la ANSES al ser designadas como relatoras en 2019 y 2020 para evaluar la necesidad de crear un nuevo peritaje colectivo sobre los horarios atípicos exceptuando el trabajo nocturno y sus efectos.

En línea con el enfoque prospectivo de Charles Gadbois, hemos y seguiremos apoyando, en el marco de nuestra participación en la ANSES, la necesidad de adoptar un enfoque sistémico y multifactorial de los efectos de estos horarios de trabajo (Barthe, 2016; ANSES, 2016). De hecho, los efectos sobre la salud de los trabajadores en horarios atípicos y en particular del trabajo nocturno, sólo tienen sentido porque los individuos están sometidos a estos horarios concretos y porque son los que trabajan durante estos horarios. Y la discordancia temporal en la que se ven envueltos los trabajadores que trabajan en horarios atípicos no puede ser explicada si se estudian solo los ritmos biológicos, o solo los sociales y/o solo los familiares. Analizar solamente las exigencias laborales y las posibilidades de regulación en el trabajo no permite comprender el equilibrio que encuentran los trabajadores en dichas situaciones de desfasaje de la jornada laboral. Del mismo modo, tener en cuenta únicamente los acuerdos conyugales, los acuerdos de custodia y otras regulaciones socio-familiares no permite comprender plenamente los efectos sobre la salud.

\begin{abstract}
"La generalización de los resultados de la investigación sobre el trabajo por turnos requiere una atención especial debido al carácter multidimensional de las situaciones del trabajo por turnos. Las dificultades encontradas sobre este punto se ponen de manifiesto al mostrar cómo se diferencian las repercusiones del trabajo por turnos en función de distintos ejes : estructura de los horarios de trabajo, cuya caracterización implica una serie de parámetros, diversidad de tareas y variabilidad de las exigencias durante la jornada, pluralidad de ritmos circadianos, diversidad de la estructura temporal de la vida fuera del trabajo, multiplicidad de niveles y ámbitos de impacto del trabajo por turnos sobre el individuo, intervención de variables moderadoras. Esta revisión conlleva sugerencias sobre cómo garantizar una evaluación más precisa del perímetro de validez de la investigación sobre el trabajo por turnos, tanto en lo que respecta al diseño de la investigación como al uso de los resultados para su aplicación" (Gadbois, 1990, p. 345, traducción libre).
\end{abstract}

Así, los modelos enumerados por Charles Gadbois en su texto - el modelo de actividad (Leplat, 2000), el modelo de las repercusiones del horario nocturno sobre la actividad (Gadbois \& Quéinnec, 1984) y el modelo del sistema de las actividades (Curie \& Hajjar, 1987) - identifican, a distintos niveles y escalas, el acoplamiento o la interdependencia entre las dimensiones "individuo - trabajo - familia", que son las dimensiones mínimas que se deben tener en cuenta al abordar el problema de los horario de trabajo.

21 Proponemos adoptar un enfoque sistémico y multifactorial para la investigación y la acción en ergonomía sobre el tiempo de trabajo. Un enfoque que identifica un conjunto de dimensiones atravesadas por el tema de los horarios atípicos, donde la actividad de trabajo y la actividad de conciliación contribuyen a mantener el equilibrio (ANSES, 2016; Barthe, 2016). Estas dimensiones retoman las identificadas por Quéinnec, Teiger y de Terssac en su libro "Repères pour négocier le travail posté " $(1992,2008)$, a saber, los horarios de trabajo, la salud, la vida social y familiar, el salario y las características del trabajo. Se complementan con otras dimensiones que tienen que ver tanto con (1) 
las características del individuo : reloj biológico, edad, cronotipo, género, resistencia a la falta de sueño, vida social y familiar, vivienda y transporte, como con (2) las características del trabajo: sistema horario, salario y primas, contenido del trabajo, factores de exigencia y posibilidades de regulación.

Figura 1 : Enfoque multifactorial y sistémico de los efectos de los horarios de trabajo (Barthe, 2016 ; ANSES, 2016)

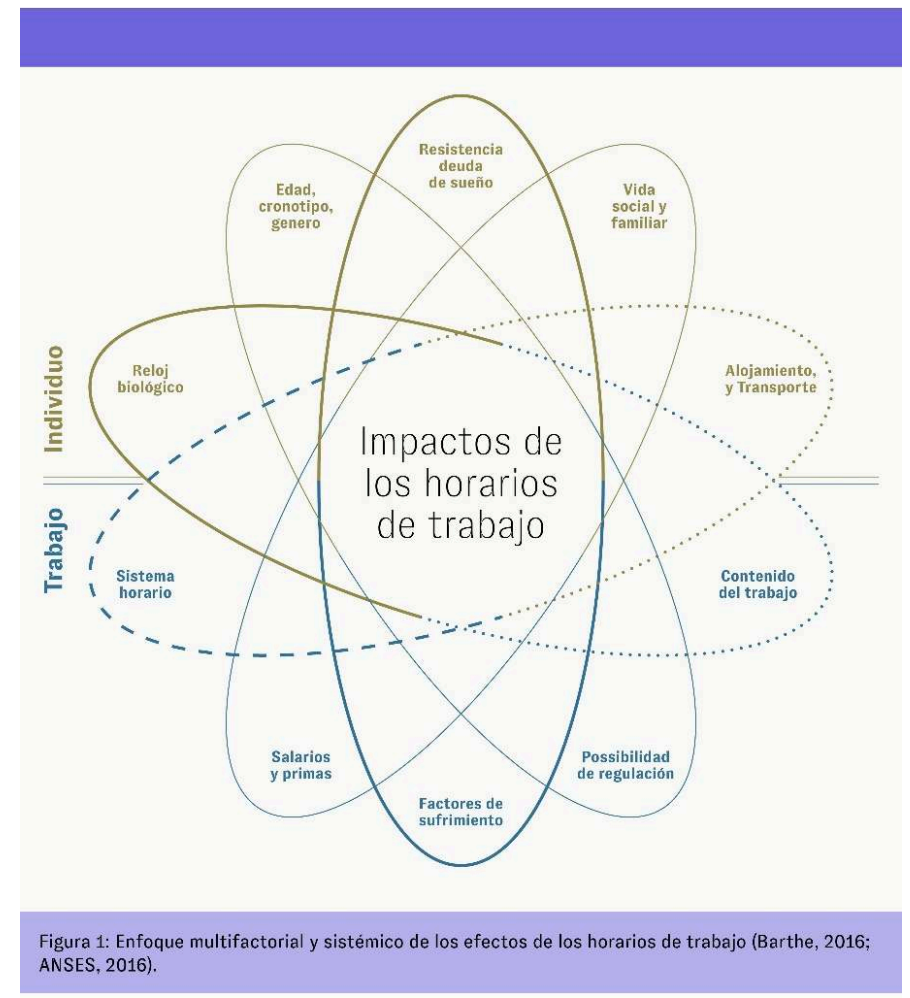

Figura 1 : Enfoque multifactorial y sistémico de los efectos de los horarios de trabajo (Barthe, 2016 ; ANSES, 2016)

Esto añade complejidad e interés en el análisis del impacto en la alteración de los ritmos fisiológicos y sociológicos causados por los horarios del trabajo por turnos y nocturno. Interés remarcado por Charles Gadbois desde sus primeros trabajos en el sector hospitalario (Gadbois, 1980). Este enfoque sistémico de los horarios de trabajo implica jugar con varias palancas de acción, combinándolas lo mejor posible para aumentar su eficacia (Barthe, 2015 ; Toupin, Barthe, \& Prunier Poulmaire, 2014).

Entre estas palancas, podemos mencionar :

- la modificación del sistema de horarios para minimizar las alteraciones circadianas y de sueño, favorecer la recuperación de la deuda de sueño del trabajador y le permita conciliar lo mejor posible su vida personal y profesional

- las acciones de apoyo de la actividad laboral y el equilibrio entre el trabajo y la vida privada identificadas,

- las acciones sobre las condiciones de trabajo y el contenido del trabajo para no aumentar los efectos de los horarios nocturnos y por turnos en los trabajadores y facilitar la gestión de la vida personal y familiar

- la acción sobre las trayectorias profesionales y la gestión de los recursos humanos con el fin de controlar la duración de la exposición de los trabajadores. 


\section{El futuro : el tiempo en perspectiva}

Si los trabajos de Charles Gadbois fueron claramente fundacionales en la materia, hoy conviene proseguirlos con un prisma similar pero seguramente enriquecido. Si bien perduran en las empresas los modelos 3x8, 4x6 y el trabajo nocturno, ya no son los únicos que deben llamarnos la atención. Hoy en día, los modelos de horarios atípicos se desarrollan más rápido de lo que la investigación científica logra comprender sus efectos.

En efecto, a diferencia de lo que ocurría hace 30 años, el trabajo en horarios atípicos refiere a un sinfín de realidades profesionales : trabajar muy temprano por la mañana y/o tarde por la noche, trabajar durante el fin de semana, trabajar largas jornadas con una pausa mayor a 3 horas o sin interrupción, trabajar toda la noche, los sábados, algunos domingos, trabajar medio tiempo, trabajar sólo cuando se le llama, etc. Algunos trabajadores combinan varias de estas realidades en la organización temporal de su trabajo. Y estas formas de trabajo afectan a una proporción cada vez mayor de trabajadores: los datos nacionales e internacionales al respecto son elocuentes. Más allá de estas exigencias de tiempo, estos trabajadores experimentan condiciones de trabajo desfavorables para su salud, claramente identificadas por las encuestas nacionales e internacionales : presión de tiempo, molestias físicas, falta de autonomía, etc. También aquí hay una gran diversidad de configuraciones posibles según las situaciones.

Entonces, si bien los conocimientos establecidos hoy sobre lo que podríamos llamar "horarios atípicos tradicionales" - horarios mayoritarios en la época del texto de Charles Gadbois- parecen estabilizados, el contexto actual exige ir más allá del trabajo por turnos y nocturno y examinar los efectos de las nuevas modalidades de los horarios atípicos. Se trata de una cuestión urgente porque estos horarios se desarrollan y se implantan rápidamente en las empresas sin conocimientos científicos sólidos en los que basarse para acompañar los cambios en curso o para sugerir las mejores condiciones posibles para preservar la salud y respetar el frágil equilibrio de los tiempos de vida.

27 El tiempo de trabajo dibuja los contornos de la vida fuera del trabajo. Pero también termina "marcando el ritmo" de toda la sociedad. En la última década, hemos asistido a una multiplicación de los ritmos de vida, incluso dentro de la pareja, la familia y más ampliamente de la ciudad. Lo que está en juego va más allá de las fronteras de la empresa, de la fábrica y de la oficina : afecta los ritmos de vida, el modelo dominante que evoca Charles Gadbois en su obra. Por lo tanto, es necesario estar alerta.

Si las investigaciones siguen siendo insuficientes, conviene en el futuro y como invitaba en su momento Charles Gadbois, establecer vínculos con otros campos disciplinarios como la psicología social y la sociología. Debemos crear las condiciones que puedan favorecer el desarrollo de esta perspectiva teórica más allá de las interacciones entre biólogos, psicólogos y sociólogos. Esto es aún más relevante si consideramos que existe una influencia recíproca de los tiempos de vida y que, -como sugieren Curie y Hajjar (1987) - los diferentes entornos y tiempos de socialización de los individuos forman un sistema compuesto por cuatro campos (profesional, familiar, personal y social) que son a la vez autónomos e interdependientes. El enfoque sistémico y multifactorial presentado en este texto invita, así, a la integración de conocimientos procedentes de otros campos disciplinarios : la cronobiología, la fisiología y las ciencias del sueño a fin 
de incluirlos en una perspectiva más bien individual de la actividad; además de los conocimientos en psicología social y sociología, impulsados por Charles Gadbois en una perspectiva socio-familiar de la actividad, conocimientos en geografía urbana que analicen los ritmos de la vida nocturna y los tiempos de las ciudades; y conocimientos de otras disciplinas como el derecho del trabajo, la demografía del trabajo, la economía o la epidemiología.

Sin embargo, nos enfrentamos a nuevos retos: las Nuevas Tecnologías de la Información y la Comunicación (NTIC), la Inteligencia artificial (IA), la robotización y la evolución irreversible de las profesiones van a cambiar aún más la situación. ¿La fábrica 4.0 hará retroceder las temporalidades nocturnas a favor de un control a distancia que no nos exija estar presentes a toda hora? ¿Quién deberá adaptar su ritmo al del otro: los humanos o los robots? Podemos imaginar o esperar que esto vendrá acompañado por ejemplo de una reducción de las exigencias, de una disminución de las horas de trabajo nocturnas.... pero nada de esto probable. No hay prácticamente ninguna razón para pensar que las nuevas formas de horarios atípicos de trabajo disminuyan. Sin embargo, no sabemos casi nada sobre sus efectos y sus consecuencias sanitarias, sociofamiliares y societarias : no disponemos de suficientes investigaciones internacionales sobre estos puntos, para responder a las demandas sociales, cada vez más apremiantes y permitir la organización de horarios de trabajo que no perjudiquen la salud ni el equilibrio de los tiempos de vida de quienes está sometidos a ellos. Sin embargo, creemos que sería útil sustentar como lo hizo Charles Gadbois, las decisiones de las empresas y las de los políticos para acompañar la expansión de estas modalidades temporales sin mayores riesgos.

Deberemos asegurarnos que no sean los más vulnerables, los que tienen un bajo nivel de calificación, los que tienen las condiciones de trabajo más duras y los horarios más difíciles, los que sigan siendo "los primeros de turno". Deberemos intentar luchar contra una sociedad que avanza a diferentes velocidades donde los ritmos son tales que los tiempos comunes se vuelvan difíciles.

31 A través de este texto, Charles Gadbois también nos invita a considerar los problemas vinculados a los marcos temporales desde un punto de vista diacrónico, a nivel de vida individual. Sugiere que los horarios atípicos de trabajo conducen a la aplicación de regulaciones en el marco de la vida personal y profesional de los trabajadores y requieren políticas de gestión coherentes y anticipadas para estos trabajadores. También denuncia esta "erosión selectiva que afecta a la población de trabajadores por turnos", lamentando que esta línea de análisis no se haya explotado más allá de los trabajos de Laville y Volkoff. También en este sentido y con la necesaria retrospectiva, la obra de Charles Gadbois es fundamental. Visionario, consideró anticipadamente que el tiempo de trabajo debe ser pensado y comprendido a escala de vida (Barthe, Garrigou, Gaudart, \& Prunier-Poulmaire, 2020), y también a escala de la carrera profesional (Prunier-Poulmaire, 2018 ; Toupin, Barthe, \& Prunier-Poulmaire, 2014), y en este sentido, los responsables de la gestión de recursos humanos tienen un papel esencial, aunque todavía están subestimados y poco dimensionados. Lo que está en juego es mucho, porque pocos factores del ámbito profesional tienen un impacto tan importante como la definición del tiempo de trabajo. El aumento de los horarios atípicos de trabajo va acompañado de importantes retos para los poderes públicos. Los temas de salud pública, de igualdad de género, el desarrollo de la política de la primera infancia, el empleo y las condiciones de trabajo se plantean más que nunca. El tiempo 
de trabajo y su organización siguen siendo, como en la época en que Charles Gadbois escribió su texto, una cuestión social de primer orden.

\section{BIBLIOGRAFÍA}

ANSES (2016). Évaluation des risques sanitaires liés au travail de nuit. Avis de l'Anses (Agence nationale de sécurité sanitaire de l'alimentation, de l'environnement et du travail). Rapport d'expertise collective. https://www.anses.fr/fr/system/files/AP2011SA0088Ra.pdf

Barthe, B. (2015). La déstabilisation des horaires de travail. In A. Thébaud-Mony, P. Davezies, L. Vogel, \& S. Volkoff (Eds.), Les risques du travail (pp. 223- 232). Paris : Éditions la Découverte.

Barthe, B. (2016). Temps de travail atypiques : désaccords temporels, des accords par l'activité. Perspectives individuelles, collectives et socio-familiales pour aménager les temps de travail. Mémoire d'Habilitation à Diriger des Recherches. Université de Toulouse 2, Toulouse.

Barthe, B., Gadbois, C., Prunier-Poulmaire, S., \& Quéinnec, Y. (2004). Travailler en horaires atypiques. In P. Falzon (Ed), Ergonomie (pp. 129-144). Paris : Presses Universitaires de France. https://doi.org/10.3917/puf.falzo.2004.01.0129

Barthe B., Quéinnec Y., \& Verdier, F. (2004). L'analyse de l'activité de travail en postes de nuit : bilan de 25 ans de recherches et perspectives. Le Travail Humain, 67(1), 41-61. https://doi.org/ $10.3917 /$ th.671.0041

Barthe, B., Garrigou, A, Gaudart, C., \& Prunier-Poulmaire, S. (2020, 18 février). Réforme des retraites et pénibilité : il faut agir sur la conception du travail. Journal le Monde. https:// www.lemonde.fr/idees/article/2020/02/17/reforme-des-retraites-et-penibilite-il-faut-agir-surla-conception-du-travail_6029810_3232.html

Costa, G., Prunier-Poulmaire, S., \& Barthe, B. (2013). Horaires de travail et société : quels constats, quelles évolutions en Europe et en France? In 48ème Congrès de la SELF-Ergonomie et société : quelles attentes? Quelles réponses? Paris : SELF.

Curie, J., \& Hajjar, V. (1987). Vie de travail, vie hors travail : la vie en temps partagé. In C. LévyLeboyer, \& J-C. Sperandio (Eds.), Traité de psychologie du travail (pp. 37-55). Paris : PUF.

Gadbois, C., \& Quéinnec, Y. (1984). Travail de nuit, rythmes circadiens et régulation des activités. Le Travail Humain, 4(3), 195-226.

Gadbois, C. (1975). L'analyse des emprises réciproques de la vie de travail et de la vie hors travail. Bulletin du CERP, 117-151.

Gadbois, C. (1980). Les exigences du travail hospitalier de nuit comme facteurs de la charge de travail. Le Travail Humain, 43(1), 19-31.

Gadbois, C. (1990). L'exacte mesure des situations de travail posté : au-delà des similitudes formelles, des réalités différentes. Le Travail Humain, 53(4), 329-345.

Gadbois, C. (1993). Impératifs des systèmes socio-techniques et temporalités humaines. In Actes du colloque de prospective : Recherches pour l'ergonomie (pp. 183-186). Université Toulouse le Mirail, Toulouse. 
Leplat, J. (2000). L'analyse psychologique de l'activité en ergonomie. Toulouse : Octarès.

Prunier-Poulmaire, S., \& Gadbois, C. (2004). Temps et rythmes de travail. In E. Brangier, A. Lancry, \& C. Louche (Eds.), Les dimensions humaines du travail. Théories et pratiques de la psychologie du travail et des organisations (pp. 181- 212). Nancy : PUN.

Prunier-Poulmaire, S., Gadbois, C., Ghéquière, A., \& De La Garza, C. (2011). Pensar la organización del tiempo de trabajo cuando la tecnología cambia : El caso del equipo de operación de una central nuclear. Laboreal, 7(2), 10-24. https://doi.org/10.4000/laboreal.7518

Prunier-Poulmaire, S., Gadbois, C., \& Volkoff, S. (1998). Combined effects of shift systems and work requirements on customs officers. Scandinavian Journal of Work, Environment and Health, 24(3), 134-140.

Prunier-Poulmaire, S. (2018, 8 février). Quand le numérique transforme l'emploi. Journal le Monde. Quéinnec, Y. Teiger, C., \& de Terssac, G. (2008). Repères pour négocier le travail posté. Toulouse : Octarès Editions

Recherches pour l'ergonomie (1993). Actes du colloque de prospective, PIR Cognisciences du CNRS. Université Toulouse le Mirail, 18-19 novembre 1993, Toulouse.

Recherches et Ergonomie (1998). Actes des deuxièmes journées "Recherches et ergonomie", Société d'Ergonomie de Langue Française (SELF), Laboratoire Travail et cognition (LTC). Université Toulouse le Mirail, 9-11 février 1998, Toulouse.

Thoemmes, J. (2000). Vers la fin du temps de travail. Paris : PUF

Toupin, C., Barthe, B., \& Prunier-Poulmaire, S. (2014). From constrained to constructed working time : Toward an enabling organization of work in rotating shifts and night shifts. In P. Falzon (Ed.), Constructive Ergonomics (pp. 65-78). Boca Raton.

Volkoff, S., Laville, A., \& Maillard, M. (1992). Age et travail : Contraintes, sélection et difficultés chez les 40-50 ans. Travail et Emploi, 54, 20-33

\section{RESÚMENES}

El texto de Charles Gadbois, publicado hace 30 años, propone, desde un enfoque prospectivo, líneas de trabajo para desarrollar la investigación en ergonomía sobre horarios atípicos de trabajo. Partiendo de la constatación que existe un desconocimiento sobre los problemas que plantea la rápida expansión de estos horarios, Charles Gadbois defendió la importancia de reunir y articular varias disciplinas para tratar esta problemática compleja, así como la necesidad de diseñar nuevos modelos que integren el de la actividad.

Desde entonces, el desarrollo de la ergonomía como ciencia interdisciplinaria y la continuación de estudios sobre los horarios atípicos de trabajo nos han llevado, en línea con lo que este autor preconizaba, a proponer un enfoque sistémico y multifactorial de la investigación y la acción sobre los horarios de trabajo. Un enfoque original que identifica un conjunto de dimensiones atravesadas por la cuestión de los horarios atípicos de trabajo, en el que la actividad laboral y la actividad de conciliación contribuyen a mantener el equilibrio.

O texto de Charles Gadbois, publicado há 30 anos, propõe, numa abordagem prospetiva, eixos de trabalho para desenvolver a investigação em ergonomia sobre os horários atípicos de trabalho. Partindo da constatação de que havia uma falta de conhecimento sobre as questões levantadas por estes horários em rápida expansão, Charles Gadbois defendeu na altura a importância de convocar e articular várias disciplinas para lidar com este problema complexo, bem como a 
necessidade de conceber novos modelos integrando o da atividade. Desde então, o desenvolvimento da ergonomia como ciência interdisciplinar e a prossecução dos trabalhos sobre os tempos de trabalho atípicos levaram-nos, na linha do que defendeu, a propor uma abordagem sistémica e multifatorial para a investigação e ação sobre os tempos de trabalho. Uma abordagem original que identifica um conjunto de dimensões atravessadas pela questão dos tempos atípicos, em que a atividade de trabalho e a atividade de conciliação contribuem para manter o equilíbrio.

Le texte de Charles Gadbois, publié il y a 30 ans, propose dans une démarche prospective, des axes de travail pour développer la recherche en ergonomie sur les horaires atypiques. Partant du constat d'un manque de connaissances sur les questions soulevées par ces horaires en pleine expansion, Charles Gadbois défendait à l'époque l'importance de convoquer et d'articuler plusieurs disciplines pour traiter de cette problématique complexe ainsi que la nécessité de concevoir de nouveaux modèles intégrant celui de l'activité. Depuis, le développement de l'ergonomie comme science interdisciplinaire et la poursuite des travaux sur les temps de travail atypiques, nous amène, dans la juste lignée de ce qu'il prônait, à proposer une approche systémique et multifactorielle pour la recherche et l'action sur les temps de travail. Une approche originale qui identifie un ensemble de dimensions traversées par la question des temps atypiques dont l'activité de travail et l'activité de conciliation participent à maintenir l'équilibre.

The article by Charles Gadbois, published 30 years ago, proposes, in a forward-looking approach, lines of work to develop research in ergonomics on atypical working times. Based on the premise that there was a lack of knowledge on the issues raised by these rapidly expanding schedules, Charles Gadbois defended the importance of calling on and articulating several disciplines to deal with this complex problem as well as the need to design new models integrating the real activity. Since then, the development of ergonomics as an interdisciplinary science and the pursuit of work on atypical working time has led us to propose a systemic and multifactorial approach for research and action on working time. An original approach that identifies a set of dimensions crossed by the issue of atypical working time, in which work activity and conciliation activity contribute to maintaining balance.

\section{ÍNDICE}

Palavras-chave: horários atípicos, ergonomia, investigação, interdisciplinaridade, tempos de trabalho

Mots-clés: horaires atypiques, ergonomie, recherche, interdisciplinarité, temps de travail

Keywords: atypical working hours, ergonomics, research, interdisciplinarity, working time

Palabras claves: horarios atípicos, ergonomía, investigación, interdisciplinariedad, tiempo

\section{AUTORES}

\section{SOPHIE PRUNIER-POULMAIRE}

https://orcid.org/0000-0002-4594-4481

Université Paris Nanterre, 200, avenue de la République, 92000 Nanterre

sophie.poulmaire@parisnanterre.fr

\section{BÉATRICE BARTHE}

Université de Toulouse ; Laboratoire LPS-DT, 5, allée A. Machado, 31058 Toulouse

beatrice.barthe@univ-tlse2.fr 